Sādhanā, Vol. 17, Part 2, June 1992, pp. 299-313. (C) Printed in India.

\title{
Approximate factorization scheme for transonic potential flow computations*
}

\author{
SUNIL KUMAR CHAKRABARTTY \\ Computational and Theoretical Fluid Dynamics Division, National \\ Aeronautical Laboratory, Bangalore 560017, India \\ MS received 15 March 1991; revised 6 April 1992
}

\begin{abstract}
The implicit approximate factorization scheme known as AF2 is investigated here for the purpose of application to the solution of twoand three-dimensional transonic full potential equations in conservative form. The artificial viscosity used by different authors has been deduced, and is discussed in detail. A second-order correction to the implicit artificial viscosity is tested for transonic flow past a Korn aerofoil at both design and off-design conditions. The inviscid transonic flow past different aerofoils, wings and wing-body configurations has been computed using the AF2 scheme and the solutions are compared with experimental and other numerical results. It is shown that the AF2 scheme is fast, and is not sensitive to grid stretching.
\end{abstract}

Keywords. Implicit schemes; approximate factorization schemes; inviscid flows; transonic flows; computational fluid dynamics.

\section{Introduction}

The computation of transonic potential flow past aerofoils, wings and bodies has reached a state of relative maturity due to the development of fast algorithms, such as the Alternating Direction Implicit (ADI) and Approximate Factorization (AF) schemes and multigrid techniques, complemented by the availability of high speed computers with vast memories. The inaccuracy introduced by the assumption of irrotationality (which is justified only for weak shocks) in potential flow computation has been accepted in order to save on the computer storage and time that would be required to obtain solutions of the more accurate Euler and Navier-Stokes equations.

For many years, Successive Line Over-Relaxation (SLOR) has proved to be a reliable but slow method for solving the compressible potential equation. The use of successive mesh refinement does hasten convergence but demands a considerable number of

\footnotetext{
* Modified version of the paper presented at CAARC (Commonwealth Advisory Aeronautical Research Council) Specialists Meeting on Computational Fluid Dynamics, held during 5-10 December, 1988, at National Aeronautical Laboratory, Bangalore.
} 
iterations. By combining a Poisson solver with a relaxation method, Jameson (1975) overcame this limitation and achieved fast convergence but this technique was limited to fairly simple computational domains.

Another fast method which has received attention is the multigrid method, applied to transonic flow problems by South \& Brandt (1976), Jameson (1979) and Chakrabartty \& Subramanian (1984). From a Fourier stability analysis it can be shown that the rate of convergence of an iteration scheme is related to grid spacing. For example, the convergence rate of a relaxation scheme becomes progressively worse as the number of grid points is increased, and a coarse grid calculation is faster. High frequency errors are associated with localized deviations from the converged solution, whereas low frequency errors reflect global discrepancies, for example an incorrect circulation. The idea of a multigrid algorithm is to reduce the error for different frequency bands at different grid levels, from fine to coarse. To make the multigrid strategy more effective, a solution method (SLOR or ADI-AF) needs to be developed first. For the complex grid spacing, as for example in a highly stretched and skewed grid or in a solution-adaptive grid, the implementation of a multigrid strategy becomes more and more difficult.

On the other hand, reduction of error in different frequency bands has been achieved successfully by proper choice of the sequence of an acceleration parameter $\alpha$ in AF schemes (Catherall 1981; Chakrabartty \& Subramanian 1986). Stability analysis for a linearized model equation by Ballhaus et al (1977) showed that the convergence rate in AF schemes depends strongly on the choice of the parameter $\alpha$. Although the precise estimation of the eigenvalues of the amplification matrix is not practical, a repeating sequence of $\alpha^{\prime}$ s is being used to reduce the error of a given band of eigenvalues. Maximum and minimum values of $\alpha$ are estimated and used to form a geometric sequence to cover the entire eigenvalue spectrum. Chakrabartty \& Subramanian (1986) have shown that this technique can be made comparable with the multigrid method. In recent works the approximate factorization scheme has been proved to be a reliable and effective technique for improving the convergence of transonic flow computations. Ballhaus et al (1977) described two factorization schemes $\mathrm{AF} 1$ and $\mathrm{AF} 2$ which led to rapid convergence when applied to the transonic small perturbation (TSP) equation. More recently, a variation of the AF2 scheme has been applied to both two- and three-dimensional transonic full potential equations by Holst (1979, 1980), Holst \& Ballhaus (1979), Holst \& Thomas (1982), Subramanian et al (1982) and Subramanian \& Chakrabartty $(1983,1986)$. These applications were limited to transonic flow past aerofoils and wings. Thomas \& Holst (1983) have modified the transonic wing analysis code TWING to suit vector computers and computed transonic flow past a wing-fuselage configuration. Chakrabartty (1990) has used the scalar version of the TWING code and, suitably generating the grids for arbitrary fuselage shape, computed transonic flow past wing-fuselage configurations on a UNIVAC-1100 computer.

On the other hand, Baker (1979) and Baker \& Forsey (1981) have proposed a new scheme named AF3, which is a modification of the AF2, to suit the non-conservative transonic full-potential equations. They claim that the AF3 scheme is faster than AF1 and $A F 2$, but it is sensitive to grid stretching. The convergence histories shown by them for a wing-body case are very slow in comparison with those for the wing-alone case. They also pointed out that the SLOR scheme, although slow, is not sensitive to grid stretching. Convergence histories of the present wing-body computations show that the AF2 scheme is not sensitive to grid stretching, which may be a plus point for AF2 over AF3. 


\section{Approximate factorization schemes}

An iterative solution procedure can be represented as:

$$
N C^{n}+\omega L \phi^{n}=0,
$$

where $C^{n}$ is the correction vector $\phi^{n+1}-\phi^{n}$ computed for cycle $n+1 . L$ is a given difference operator to be operated on an unknown potential $\phi$ and $L \phi^{n}=R^{n}$ is usually known as a residual at the $n$th level. $\omega$ is the relaxation parameter. The operator $N$ determines the type of iteration procedure and therefore determines the rate at which the iteration procedure converges. In approximate factorization schemes $N$ is written as a product of two or more factors,

$$
N=N 1 \cdot N 2 \cdot N 3 \ldots,
$$

each of which is restricted to a form leading to equations that can be easily solved. The added flexibility resulting from the use of several factors allows $L$ to be more closely approximated by $N$. Moreover, algorithms can be constructed along these lines in such a way that the final $N$ operator is fully implicit, so that every grid point is influenced by every other grid point during eack cycle.

For simplicity let us consider a linear two-dimensional difference operator

$$
L(\phi)=(A \vec{\delta} x \bar{\delta} x+B \vec{\delta} z \bar{\delta} z) \phi=0
$$

$A$ and $B$ are not assumed to be constants so that (2) can be taken to represent the discretized form of a flow equation (e.g. TSP or full potential) with lower-order and cross derivative terms neglected. For this case, $N$ can be written as the product of two factors, for example, the simplest form of the AF1 scheme is

$$
(\alpha-A \vec{\delta} x \bar{\delta} x)(\alpha-B \vec{\delta} z \bar{\delta} z) C^{n}=\alpha \omega L \phi^{n},
$$

where $\alpha$ is an acceleration parameter whose value needs careful selection, in order to achieve rapid convergence. The AF1 scheme was not found suitable for supercritical flows and an AF2 scheme

$$
(\alpha-A \Delta x \vec{\delta} x)(\alpha \bar{\delta} x / \Delta x-B \vec{\delta} z \overleftarrow{\delta} z) C^{n}=\alpha \omega L \phi^{n},
$$

where $\Delta x$ is the step length in the $x$-direction, was used successfully for flows with supersonic regions. Baker (1979) proposed AF3 scheme in polar co-ordinates as

$$
(-\alpha A \Delta r \vec{\delta} r-B \bar{\delta} \theta \bar{\delta} \theta)(\alpha+\bar{\delta} r / \Delta r) C^{n}=\alpha \omega L \phi^{n},
$$

which is similar to AF2 but with the factors reversed and $A$ and $B$ placed inside the first factor so that they need be calculated only during the first sweep. These three approximate factorization schemes have been studied in detail and a comparative assessment was made on the basis of numerical results for a variety of transonic flow problems by Baker (1979) using polar co-ordinates.

\section{Full-potential formulation}

Three-dimensional, inviscid, transonic full-potential equation can be written in strong conservation law form as

$$
\left(\rho \phi_{x}\right)_{x}+\left(\rho \phi_{y}\right)_{y}+\left(\rho \phi_{z}\right)_{z}=0
$$


with

$$
\rho=\left[1-\{(\gamma-1) /(\gamma+1)\}\left(\phi_{x}^{2}+\phi_{y}^{2}+\phi_{z}^{2}\right)\right]^{1 /(\gamma-1)} .
$$

The density, $\rho$ and velocity components $\left(\phi_{x}, \phi_{y}\right.$ and $\left.\phi_{z}\right)$ are non-dimensionalized by the stagnation density and the critical sound speed, respectively. $x, y$ and $z$ are Cartesian coordinates in the streamwise, spanwise and vertical directions respectively and $\gamma$ is the ratio of specific heats. The two-dimensional form of (6) can be simply obtained by dropping all $y$-derivative terms from (6). Equation (6) has been solved in a transformed $(\xi=\xi(x, y, z), \eta=\eta(x, y, z)$ and $\zeta=\zeta(x, y, z))$ co-ordinate system $(\xi, \eta, \zeta)$ where it maintains the strong conservation law form as

with

$$
(\rho U / J)_{\xi}+(\rho V / J)_{\eta}+(\rho W / J)_{\zeta}=0
$$

$$
\rho=\left[1-\{(\gamma-1) /(\gamma+1)\}\left(U \phi_{\xi}+V \phi_{\eta}+W \phi_{\zeta}\right)\right]^{1(\gamma-1)},
$$

where $U, V$ and $W$ are the contravariant velocity components along $\xi, \eta$ and $\zeta$ directions, respectively, and $J$ is the Jacobian of transformation. Details of the spatial differencing and AF2 scheme for two-dimensional flow are given by Holst (1979) and Holst \& Ballhaus (1979) and those for three-dimensioanl flows are given by Holst (1980) and Holst \& Thomas (1982).

\section{Artificial viscosity in AF2 scheme}

The formulation of finite difference approximations for transonic flow computation is based on an idea introduced by Murman \& Cole (1971). This uses central difference formulas in the subsonic zone, where the flow equation is elliptic, and upwind difference formulas in the supersonic zone, where it is hyperbolic. Thus the numerical scheme has a directional bias. This corresponds to the upwind region of dependence of the flow in the supersonic zone, and also serves the purpose of enforcing the entropy condition that discontinuous expansions must be excluded.

The dominant term in the discretization error introduced by the upwind differencing acts like an artificial viscosity. Instead of using a switch in the differencing scheme to introduce a viscosity, we can explicitly add a viscosity which produces an upwind bias in the difference scheme at supersonic points. In the Murman \& Cole (1971) scheme the error introduced by replacing central difference by the backward difference for $\phi_{x x}$ is $\Delta x \phi_{x x x}$. So,

$$
\bar{\phi}_{x x}=\phi_{x x}-\Delta x \phi_{x x x}
$$

The last term is an artificial viscosity term which tends to zero as $\Delta x$ tends to zero. Now if we write

where

$$
\phi_{x x}=\left.\phi_{x x}\right|_{\text {central }}-\mu \Delta x \phi_{x x x}
$$

$$
\mu= \begin{cases}0, & \text { for subsonic flow, } \\ 1, & \text { for supersonic flow. }\end{cases}
$$

Thus we get the central difference in subsonic and the backward difference in supersonic regions. $\mu$ is called the switching parameter. 
Considering the two-dimensional form of (6) and introducing

we get

$$
a^{2}=[(\gamma-1) / 2] \rho^{\gamma-1},
$$

$$
\rho_{x}=-\left(\rho / a^{2}\right)\left(\phi_{x} \phi_{x x}+\phi_{z} \phi_{x z}\right)
$$

or

$$
\frac{\partial}{\partial x}\left(\rho \phi_{x}\right)=\rho \phi_{x x}\left(1-\left(\phi_{x}^{2} / a^{2}\right)\right)-\left(\rho / a^{2}\right) \phi_{x} \phi_{z} \phi_{x z}
$$

From (12)

Therefore

$$
\rho \phi_{x x}=-\left\{\left(a^{2} \rho_{x} / \phi_{x}\right)+\rho\left(\phi_{z} / \phi_{x}\right) \phi_{x z}\right\}
$$

$$
\frac{\partial}{\partial x}\left(\rho \phi_{x}\right)=\rho_{x} \phi_{x}\left(1-\left(a^{2} / \phi_{x}^{2}\right)\right)-\rho\left(\phi_{z} / \phi_{x}\right) \phi_{x z}
$$

Similarly

$$
\frac{\partial}{\partial z}\left(\rho \phi_{z}\right)=\rho_{z} \phi_{z}\left(1-\left(a^{2} / \phi_{z}^{2}\right)\right)-\rho\left(\phi_{x} / \phi_{z}\right) \phi_{x z}
$$

or

$$
\frac{\partial}{\partial z}\left(\rho \phi_{z}\right)=\rho \phi_{z z}\left(1-\left(\phi_{z}^{2} / a^{2}\right)\right)-\left(\rho / a^{2}\right) \phi_{x} \phi_{z} \phi_{x z}
$$

Adding (13) and (17) we get

$$
\begin{aligned}
\frac{\partial}{\partial x}\left(\rho \phi_{x}\right)+\frac{\partial}{\partial z}\left(\rho \phi_{z}\right)=\left(1-\left(\phi_{x}^{2} / a^{2}\right)\right) \rho \phi_{x x}- \\
-2 \frac{\rho}{a^{2}} \phi_{x} \phi_{z} \phi_{x z}+\left(1-\left(\phi_{z}^{2} / a^{2}\right)\right) \rho \phi_{z z}
\end{aligned}
$$

and adding (15) and (16) we get

$$
\begin{aligned}
\frac{\partial}{\partial x}\left(\rho \phi_{x}\right)+\frac{\partial}{\partial z}\left(\rho \phi_{z}\right)= & \rho_{x} \phi_{x}\left(1-\left(a^{2} / \phi_{x}^{2}\right)\right)- \\
& -\rho \phi_{x z}\left(\left(\phi_{x}^{2}+\phi_{z}^{2}\right) / \phi_{x} \phi_{z}\right)+\rho_{z} \phi_{z}\left(1-\left(a^{2} / \phi_{z}^{2}\right)\right) .
\end{aligned}
$$

Since we are interested in upwinding only in the $x$-direction, the first term of the right hand side of (18) and (19) would be upwinded.

The terms are (i) $\mu \phi_{x x}$ where $\mu=\rho\left(1-\phi_{x}^{2} / a^{2}\right)$ has been used by Jameson (1978) and (ii) $v \rho_{x} \phi_{x}$ where $v=\left(1-a^{2} / \phi_{x}^{2}\right)$ has been used by Holst (1979). The above analysis shows that these two expressions are the same. Holst introduced the artificial viscosity term implicitly to modify the density which can be deduced as an error introduced due to backward differencing. He used the discretization as

$$
\frac{\partial}{\partial x}\left(\rho \phi_{x}\right)=\bar{\delta} x\left(\hat{\rho}_{i+1 / 2} \bar{\delta} x \phi_{i}\right)
$$

where

$$
\hat{\rho}_{i+1 / 2}=\left(1-v_{i}\right) \rho_{i+1 / 2}+v_{i} \rho_{i-1 / 2} .
$$

In a similar way a second-order artificial viscosity has been introduced by 
Chakrabartty \& Subramanian (1985) by defining

$$
\hat{\rho}_{i+1 / 2}=\left(1-v_{i}\right) \rho_{i+1 / 2}+v_{i} \rho_{i-1 / 2}+\lambda v_{i-1}\left(\rho_{i-1 / 2}-\rho_{i-3 / 2}\right),
$$

where $\lambda$ is a switching factor from first order to second order. For $\lambda=0$ it gives the first order and for $\lambda=1$ it gives the second order artificial viscosity. For stability the value of $\lambda$ should be small near the shock region and it should be of the order of one near the smooth supersonic region for better accuracy.

\section{Results and discussions}

The effect of second-order artificial viscosity (22) has been studied first. Supercritical Korn aerofoil has been considered to test the improvement in accuracy of the code STAIR2 (modified version of Holst's TAIR code with second-order correction) over TAIR. This is a typical aerofoil where one expects a shock-free supercritical solution at design conditions. The pressure distributions obtained by the modified code STAIR 2 and by other codes like TAIR and STAMAD (Multigrid with ADI) have been compared in figure 1. An almost shock-free solution has been obtained by the STAIR 2 code. The same number of grid points and the same convergence criterion have been used in all the computations. Results computed by Chakrabartty \& Subramanian $(1984,1986)$ using the code STAMAD (Multigrid with ADI) predicted stronger shock even with secondorder correction. Figure 2 shows a comparison of pressure distributions on the same aerofoil at off-design conditions where the experimental results of Kacprzynski \& Ohman (1979) show a double shock. Only the present STAIR2 code could predict the double shock behaviour. Figure 3 shows a comparison of convergence history for an NACA-0012 aerofoil. This shows that the AF2 factorization scheme can be made comparable to the multigrid method with respect to the rate of convergence if a proper choice of the sequence of the acceleration parameter $\alpha$ can be made. In the present case, the number of elements in the sequence of $\alpha$ was taken to be eight with its lowest and highest values equal to 0.07 and 1.5, respectively. Figure 4 shows another comparison of pressure distribution on the CAST-7 aerofoil with experimental results. In all the cases studied for two-dimensional flow the present code STAIR2 predicted more accurate solutions than its original counterpart TAIR.

For three-dimensional flow past the wings, figure 5 shows a comparison of pressure distributions at different span stations on an ONERA-M6 wing at $M \infty=0.84$ and

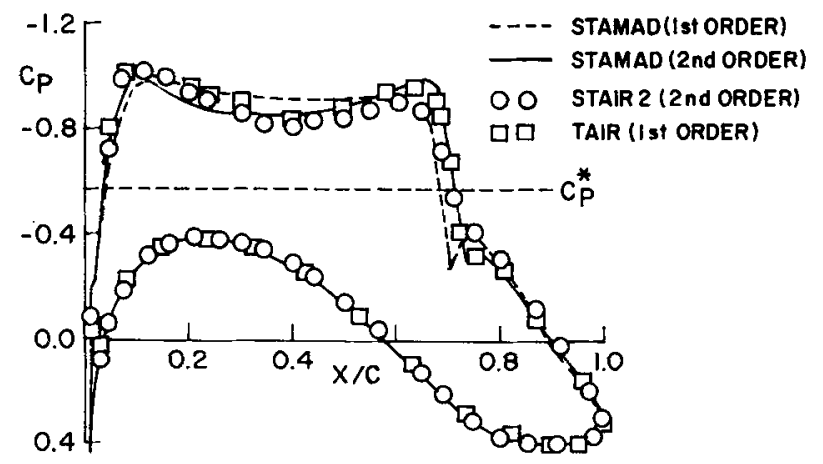

Figure 1. Pressure distribution on Korn aerofoil, at $M_{\propto}=0.75$, $x=0^{\circ}$. 




Figure 2. Pressure distribution on Korn aerofoil, at $M_{\infty}=0.75$, $\alpha=-0.5^{\circ}$.

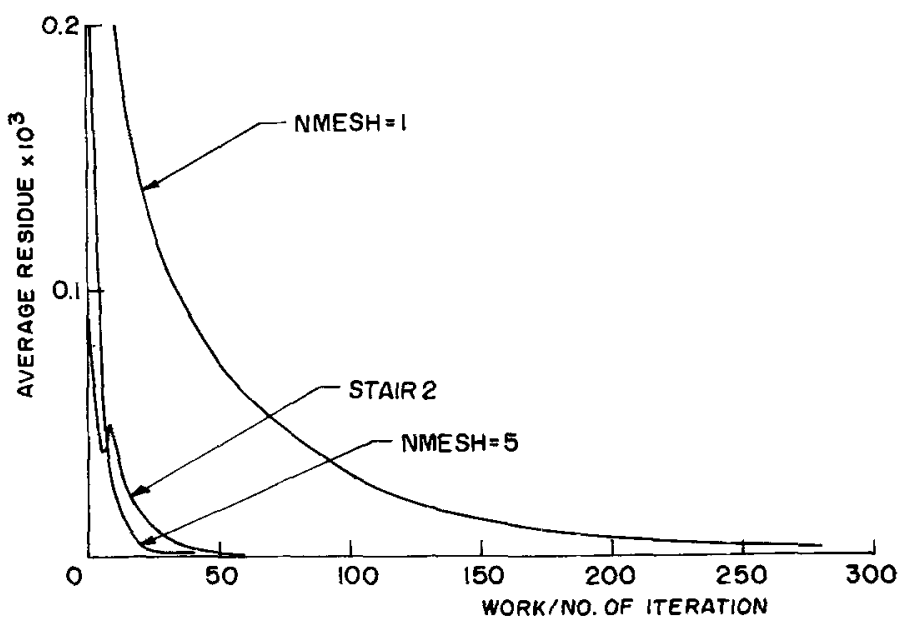

Figure 3. Comparison of convergence histories for NACA-0012 aerofoil at $M_{\infty}=0.80, \alpha=0^{\circ}$.

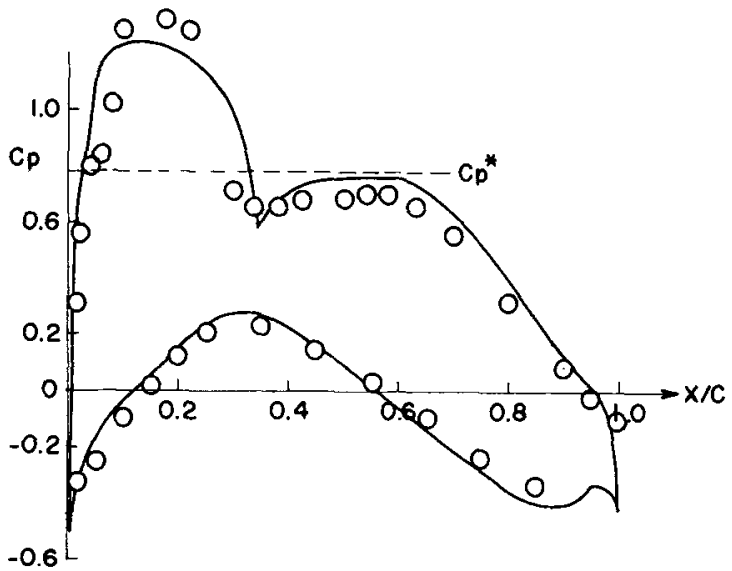

\begin{tabular}{|l|l|c|}
\hline & PRESENT & $\begin{array}{c}\text { EXPT } \\
\text { DFVLR-TWB }\end{array}$ \\
\hline & - & 0 \\
$M \infty$ & 0.70 & 0.701 \\
$\alpha$ & $0.5^{\circ}$ & $2.0^{\circ}$ \\
$N S R$ & 81 & - \\
$C L$ & 0.7527 & 0.7028 \\
$C D$ & -0.0001 & 0.0124 \\
$C M$ & -0.1447 & -0.1087 \\
ITN & 64 & \\
\hline
\end{tabular}

Figure 4. Surface pressure distribution on CAST-7 airfoil. 



Wing characteristics ONERA-M6 $M_{\alpha}=0.84$ $x=3 \cdot 06^{\circ}$

\begin{tabular}{lcc}
\hline & SFLO22 & STWING \\
\hline Grid size & $96 \times 12 \times 16$ & $89 \times 25 \times 18$ \\
$C_{L}$ & 0.2837 & 0.29356 \\
$C_{D}$ & 0.0190 & 0.0073 \\
Pitch $C_{M}$ & -0.2161 & \\
Roll $C_{M}$ & 0.2581 & \\
Yaw $C_{M}$ & 0.0018 & \\
CPU time in & 2 hours & 7 hours \\
$\quad$ UNIVAC 1100/60 & (approx.) & (approx.) \\
\hline
\end{tabular}

Figure 5. Pressure distribution on ONERA-M6 wing, at $M_{\infty}=0.84, \alpha=3.06^{\circ}$. (a) $20 \%$, (b) $40 \%$, and (c) $90 \%$ of the semi-span.

angle of attack $\alpha=3.06$. The present code STWING, which solves transonic full potential equations in conservative form using the AF2 scheme, predicted shock position better than SFLO22, which solves transonic full potential equation in non-conservative form using the SLOR method (Jameson 1975), with respect to the experimental values (AGARD 1979). Overall comparison with experiment is good. Aerodynamic characteristics of the wing obtained by the two codes have also been compared and shown in the table (figure 5). Figure 6 shows the comparison of pressure distributions on a 




(a)

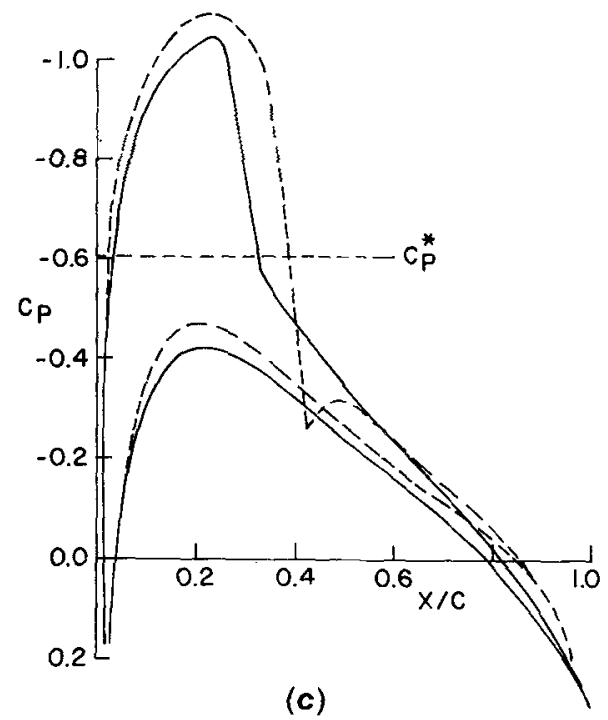

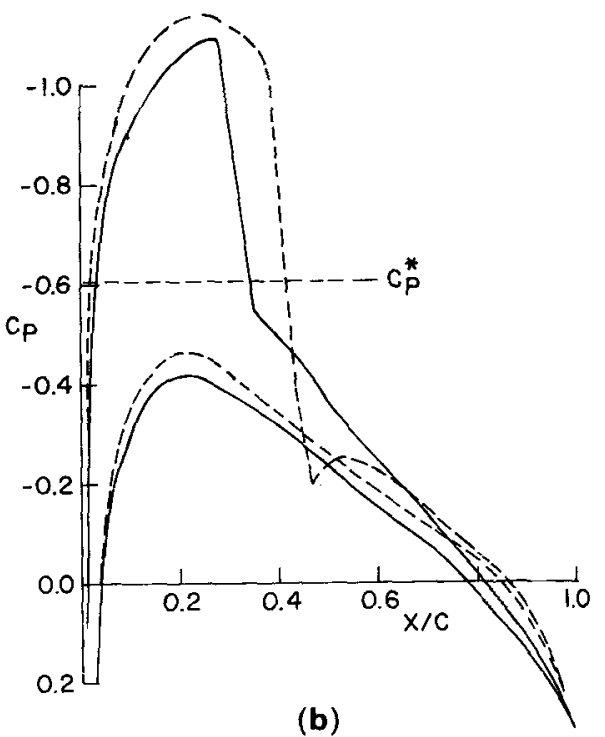



(d)

Figure 6. Pressure distribution on rectangular wing of aspect ratio $=6$, with NASA-0012 airfoil, at $M_{\infty}=0.75, \alpha=2^{\circ}$. SFLO22 (non-conservative FP); -- STWING (conservative FP); $\Delta$ upper surface, $\nabla$ lower surface, experimental results for 2-D flow. Span station $=0.0$ (a), 0.9 (b), 1.8 (c) and 2.7 (d).

rectangular wing with an NACA 0012 aerofoil. Here, the STWING code predicts stronger shock than the SFLO22 code and the experimental (two-dimensional) shock position falls in between.

A wing-body configuration, wing-A with body-B2 (figure 7), has been considered next as a standard AGARD test case. Three cases have been computed for different free-stream Mach numbers and angles of attack and shown in figures 8, 9 and 10. In figure 8 the results have been compared with experimental AGARD (1979) values and the numerical results of Baker \& Forsey (1981). The pressure distributions for the 


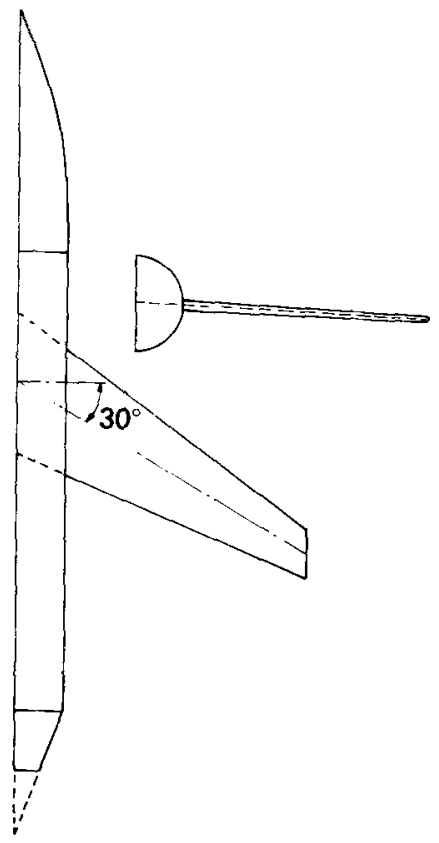

Figure 7. Wing-A and body-B2 geometry.

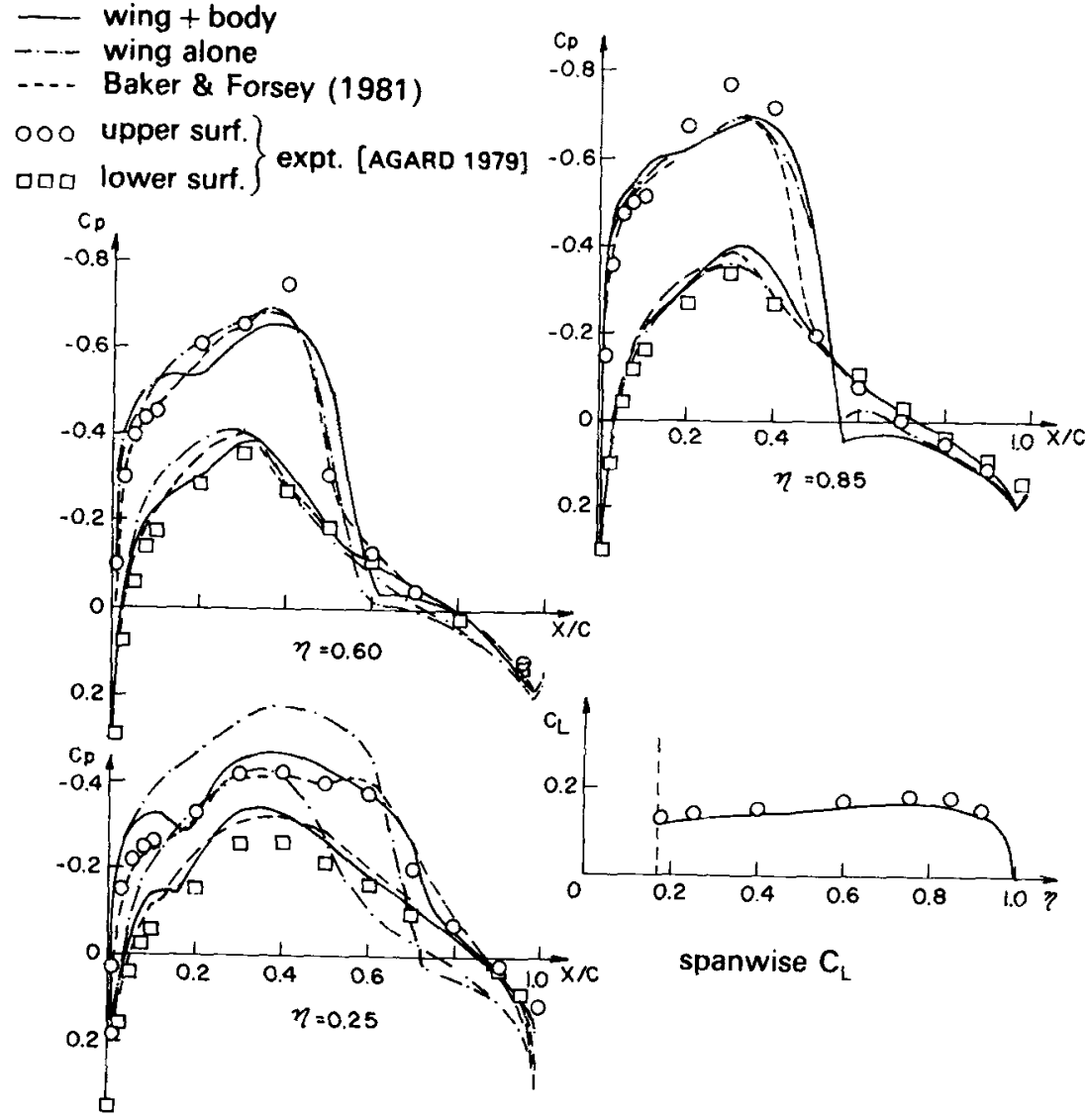

Figure 8. Pressure distribution on RAE-wing-A + body-B2, at different span stations at $M_{\infty}=0.90, \alpha=1^{\circ}$. 

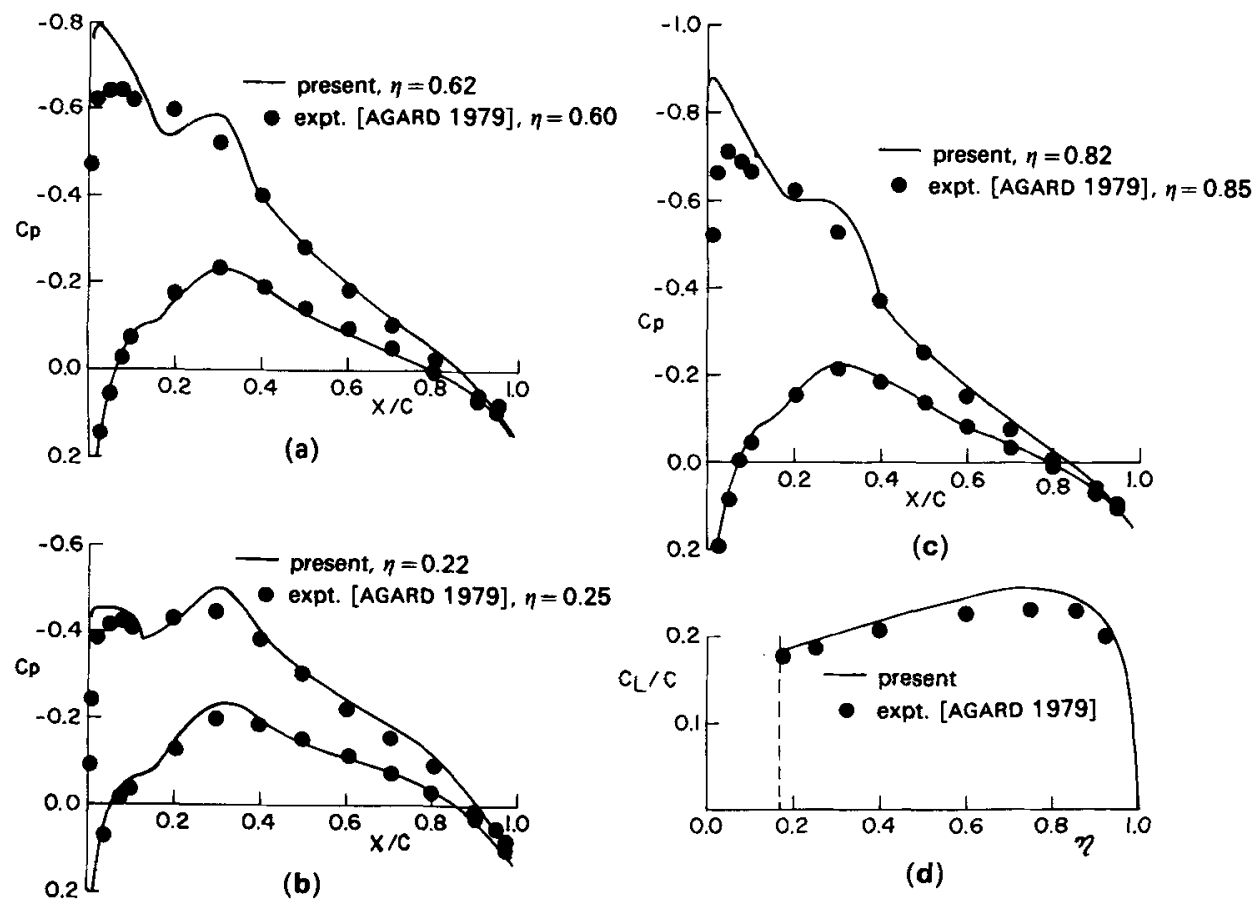

(d)

Figure 9. Pressure distribution at different span stations, (a), (b), (c) and spanwise lift distribution, (d), for transonic flow past wing- $\mathrm{A}+$ body-B2, at $M_{\infty}=0.80$,

wing-alone case have also been shown there with the same number of grid points $(89 \times 27 \times 20)$. It is apparent by comparison that the effect of the fuselage is prominent near the wing-body junction and is minimum near the tip. The span-wise $C_{L}$ distribution has also been shown in the same figure with good comparison with experiments. A two-dimensional grid generation code which solves Laplace equations in two directions has been used here to generate grids at different span-stations and then these are stretched properly to fit the fuselage (Chakrabartty 1990), to get an $\mathrm{O}-\mathrm{H}$ type three-dimensional grid. All the three cases computed for this example show that the present code STWING with the present grid generation technique gives reasonable pressure distribution and predicted good $C_{L}$ distribution in comparison with experimental results. The next example considered is a DFVLR wing-body model studied by Holst \& Raman (1988). Computed pressure distributions (figure 11), show good overall agreement with the experimental results. In all cases, the higher suction predicted by the theory near the leading edge is expected because of its inviscid approximation.

Convergence histories for the first wing-body configuration have been shown in figures 12 and 13 for two different cases taking the same number of grid points for wing-alone and wing-body combinations. It is apparent from the figures that the rate of convergence of the AF2 scheme does not depend on the grid stretching, which is an important observation for this scheme in contrast to the AF3 scheme. It can be concluded from the present investigation that the approximate factorization (AF2) scheme can give better accuracy and faster convergence if the sequence of acceleration parameters can be chosen properly with second-order accurate artificial viscosity. 

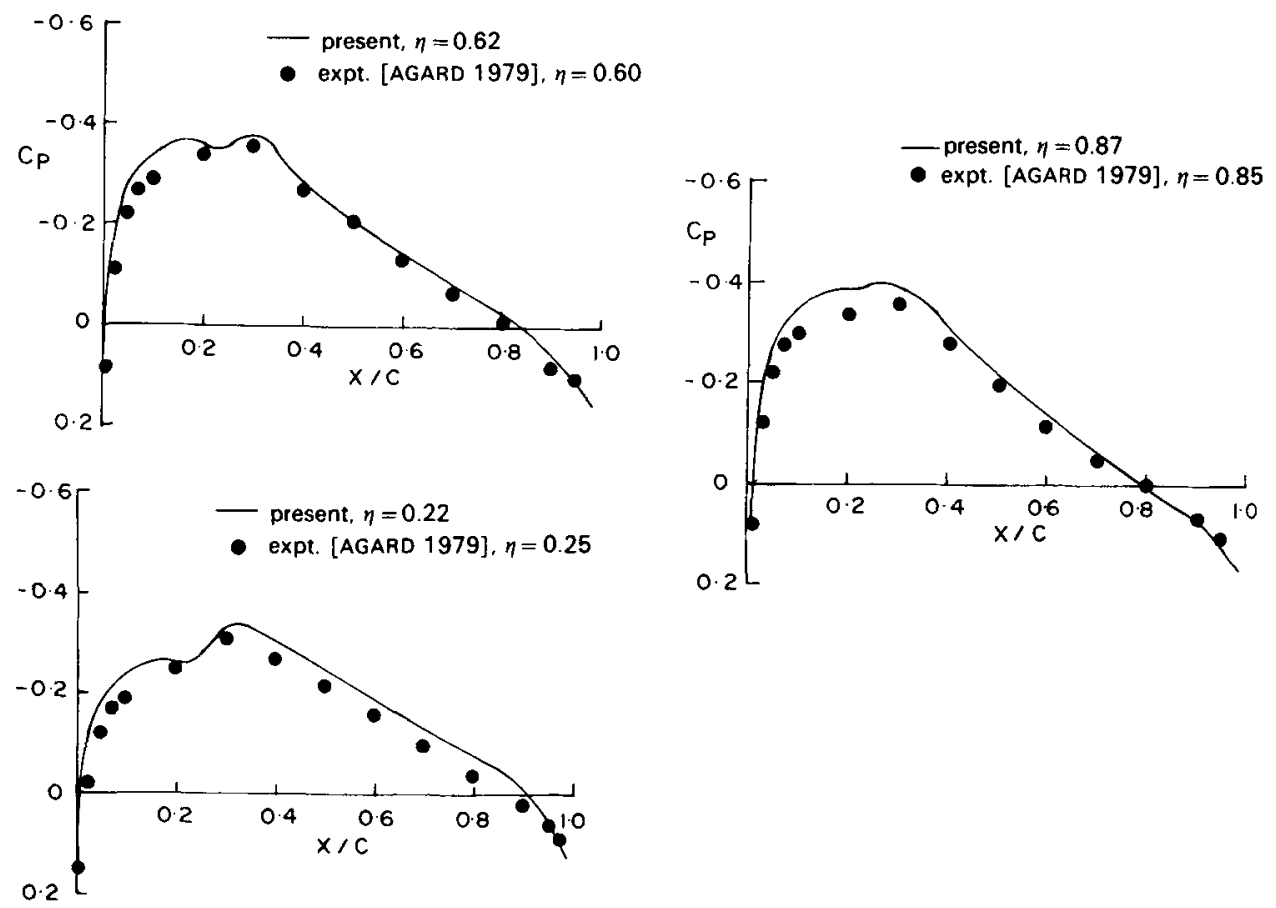

Figure 10. Pressure distribution at different span stations, for transonic flow past RAE-wing-A + body-B2, at $M_{\infty}=0.80, \alpha=0^{\circ}$.
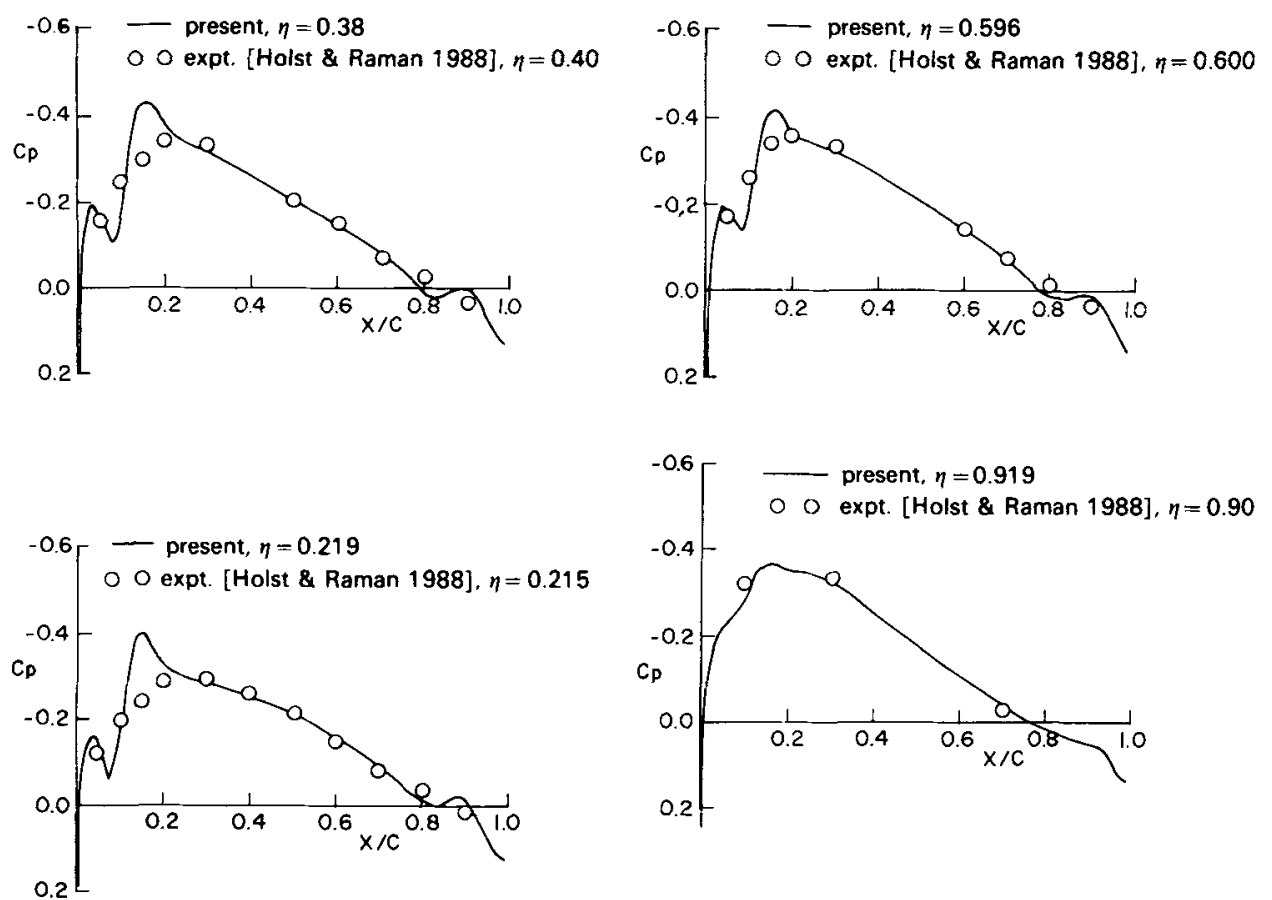

Figure 11. Pressure distribution on a DFVLR wing-body model at different span stations, at $M_{\infty}=0.70, \alpha=0^{\circ}$. 


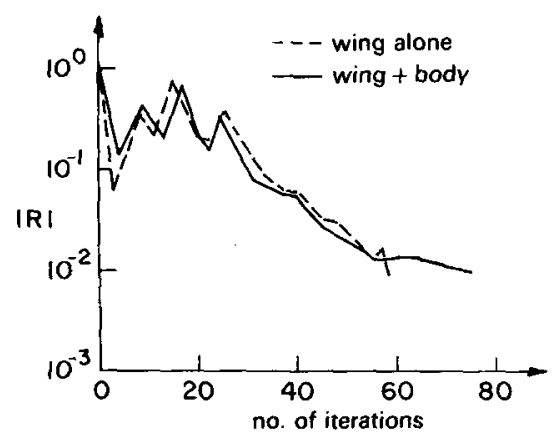

Figure 12. Convergence history for RAEwing- $\mathrm{A}+$ body-B2 at $M_{\infty}=0.90, \alpha=1^{\circ}$, $(89 \times 25 \times 20)$ grids.

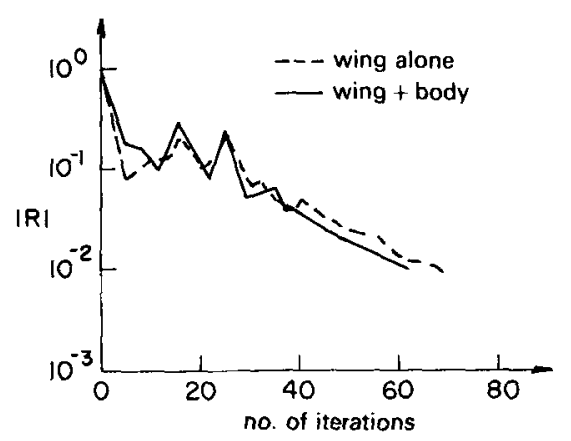

Figure 13. Convergence history for RAEwing-A + body-B2 at $M_{\infty}=0.80, \quad \alpha=2^{\circ}$, $(89 \times 25 \times 20)$ grids.

This work was partially supported by a grant-in-aid project of the Aeronautical Research and Development Board, Ministry of Defence, Government of India. The author takes this opportunity to thank Dr N R Subramanian for his continuous encouragement to complete this work.

\section{List of symbols}
$a$
$A, B$
velocity of sound, defined in (11);
$C$ coefficients, defined in (2);
correction vector, defined in (1);
$C_{L}, C_{D}, C_{M}, C_{P}$
$J$
$L$
lift, drag, moment and pressure coefficients;
Jacobian of transformation;
$M$
$N$
$N S P$
given difference operator;
Mach number;
operator defining the type of iteration procedure;
$N 1, N 2, N 3 \ldots$ number of supersonic points;
$R$
$x, y, z$
factors of the operator $N$;
residual;
Cartesian coordinate system representing chordwise, spanwise and
$r, \theta$
vertical directions, respectively;
$U, V, W$
polar coordinate system in two dimensions;
contravariant velocity components along $\zeta, \eta$ and $\xi$ directions, respectively;
$\alpha$
acceleration parameter for ADI (AF) schemes/also angle of attack;
$\omega$
$\frac{\varphi}{\delta} x, \bar{\delta} z$
$\vec{\delta} x, \vec{\delta} z$
$\vec{\delta} r, \bar{\delta} \theta, \bar{\delta} \theta$
relaxation parameter used in (1);
unknown velocity potential;
backward difference operators in $x$ and $z$ directions, respectively; forward difference operators in $x$ and $z$ directions, respectively;

$\Delta x, \Delta r$
$\rho$
$\gamma$
$\xi, \eta, \zeta$ forward difference operator in $r$ and forward and backward difference operator in $\theta$ directions, respectively, in polar coordinates; increments in $x$ and $r$ directions, respectively; density of the fluid; ratio of the specific heats at constant pressure and volume; transformed curvilinear coordinate systems; 
$\mu$

$v$

$\lambda$

\section{Super'scripts}

$n$

*

\author{
Subscripts \\ $i$ \\ $x, \ldots, \xi, \ldots$ \\ $\infty$
}

\section{References}

switching parameter in (10) or coefficient of artificial viscosity term; coefficient of artificial compressibility term; switching factor from first-order to second-order artificial compressibility term.

level of iteration; critical value.

value to be evaluated at ith grid point; derivatives with respect to the variables; free-stream value.

AGARD 1979 Experimental data base for computer program assessment, AR-138

Baker T J 1979 A fast implicit algorithm for the non-conservative potential equation, Open forum presentation, AIAA 4th Computational Fluid Dynamics Conference, Williamsburg, vA

Baker T J, Forsey C R 1981 A fast algorithm for the calculation of transonic flow over wing/body combinations, AIAA 5th Computational Fluid Dynamics Conference, Palo Alto, California, Paper no. AIAA-81-1015

Ballhaus W F, Jameson A, Albert J 1977 Implicit approximate factorization schemes for the efficient solution of steady transonic flow problems, AIAA Paper 77-634

Catherall D 1981 Optimum approximate factorization schemes for 2D steady potential flows, AIAA 5th Computational Fluid Dynamics Conference, Palo-Alto, California, AIAA-81-1018

Chakrabartty S K, Subramanian N R 1984 Transonic potential flow computations by multigrid method, NAL-FM-TM-84-12

Chakrabartty S K, Subramanian N R 1986 A comparative study of approximate factorization scheme with multigrid method for transonic flow computation, Proc. of $3 r d$ Asian Congress of Fluid Mechanics, Tokyo

Chakrabartty S K 1990 Computation of transonic potential flow past wing-body configurations. Acta Mech. 81: 201-209

Chakrabartty S K, Subramanian N R 1985 A second order accurate implicit scheme for conse vative full potential equation in transonic flow. Proc. of 37 th $A G M$ of the Aero. Soc. of India. Calcutta

Holst T L 1979 An implicit algorithm for conservative transonic full potential equation using arbitrary mesh. AIAA J. 17: 1039-1045

Holst T L 1980 Fast conservative algorithm for solving the transonic full potential equation. AIAA J. 18: 1431-1439

Holst T L, Ballhaus W F 1979 Fast conservative scheme for the full potential equation applied to transonic flows, NASA TM 78469. Also AIAA J. 17: 145-152

Holst H, Raman K S 1988 2-D adaption of 3-D testing. DEVLR-29112-88 A 03

Holst T L, Thomas S 1982 Numerical solution of transonic wing flow fields, AIAA 20th Aerospace Science Meeting, Orlando, Florida, AIAA-81-0105

Jameson A 1975 Accelerated iteration scheme for transonic flow calculations using fast Poisson solvers, ERDA Report COO-3077-82, New York University

Jameson A 1978 Transonic flow calculations. Numerical methods in fluid dynamics (eds) H J Wirtz, J J Smolderen (Washington, New York, London: Hemisphere)

Jameson A 1979 Acceleration of transonic potential flow calculations on arbitrary meshes by multigrid method, AIAA Paper 79-1458 
Kacprzynski J J, Obman L H 1979 Wind tunnel project report $5 \times 5 / 0054$, High Speed Aerodynamics Section, Ottawa

Murman E M, Cole J D 1971 Calculation of plane steady transonic flows. AIAA J. 9: 114-121

South J C, Brandt A 1976 The multigrid method: fast relaxation for transonic flows. Advances in Engineering Sciences, NASA-CP-2001, Vol. 4, pp. 1359-1369, NASA

Subramanian N R, Chakrabartty S K 1983 Computation of inviscid transonic flow past aerofoils using body-fitted co-ordinates. Proc. of 35th AGM of the Aero. Soc. of India. Bombay

Subramanian N R, Chakrabartty S K 1986 Transonic potential flow computations around finite wings. J. Aero. Soc. India 38: 55-57

Subramanian N R, Holst T L, Scott D T 1982 Recent application of transonic wing analysis computer code TWING, NASA-TM-84283

Thomas S D, Holst T L 1983 Numerical computation of transonic flow about wing-fuselage configurations on a vector computer. AIAA 21 st Aerospace Science Meeting, Reno Nevada, AIAA-83-0439 